\title{
Influence of pump noise and modulation on in-fiber amplification of broadband pulses
}

K. Gürel, I. L. Budunoğlu, Ç. Şenel, P. P. Paltani, and F. Ö. Ilday

Physics Department, Bilkent University, Çankaya, Ankara 06800,

ilday@bilkent.edu.tr

\begin{abstract}
We investigate experimentally and theoretically the coupling of pump laser modulation and noise fluctuations to the output power of a fiber amplifier for broadband pulse trains using the modulation transfer function approach.

OCIS codes: $060.2320,320.7090,060.3510$.
\end{abstract}

Rapid progress is being reported for the development of low-noise, high-power fiber laser-amplifier systems. However, despite its clear importance, intensity noise of fiber amplifiers has not received much attention. Recently, we reported a systematic study of intensity noise of mode-locked fiber oscillators, operating in different modelocking regimes and with respect to the key laser parameters [1]. An important conclusion of this study was that, in general, there is a trade-off between the noise level of the oscillator and the highest pulse energy that can be extracted and the optimal route to low-noise high-power operation could be to operate the oscillator at moderate power levels and use external in-fiber amplification. Thus, it is important to determine the conditions for which noise added during amplification is minimized. In addition, intentional modulation of pump power is commonly used in carrier-envelope phase stabilization of fiber frequency combs, where the highest modulation frequency that can be transferred to the signal ultimately limits the bandwidth of the stabilization loop. Both of these considerations motivate our investigation of the dynamics of transfer of pump modulations to signal power.

The experimental setup is shown in Fig. 1. As seed oscillator, we use either a Yb-doped fiber laser operating in the similariton mode with $35 \mathrm{MHz}$ of repetition rate and central wavelength of $1030 \mathrm{~nm}$ or another $\mathrm{Yb}$-doped fiber laser operating in the all-normal dispersion mode with $43 \mathrm{MHz}$ of repetition rate and central wavelength of $1050 \mathrm{~nm}$. The seed signal is then split by a coupler, where one port is directed to a high-resolution power meter and the other port is fiber-coupled to a forward-pumped fiber amplifier with relatively low-doped $120 \mathrm{~cm}$-long $\mathrm{Yb}$ fiber with absorption peak of $200 \mathrm{~dB} / \mathrm{m}$ at $976 \mathrm{~nm}$.

The modulation transfer function (MTF) describing coupling of modulation of pump to the amplified pulse train is measured by modulating the pump power over a frequency range from $1 \mathrm{~Hz}$ to $250 \mathrm{kHz}$ with a signal generator. The MTF is obtained via the same method as in [3]. Here, the measurement is fully automated; a computer controls the signal generator, which modulates the pump diode through its driver and a Fourier-transform-based low-noise and high-dynamic-range baseband analyzer (Rohde \& Schwarz, Audio Analyzer UPV) with bandwidth of $250 \mathrm{kHz}$. The amplitude fluctuations or noise of the amplified pulse train is characterized using the standard technique [2].

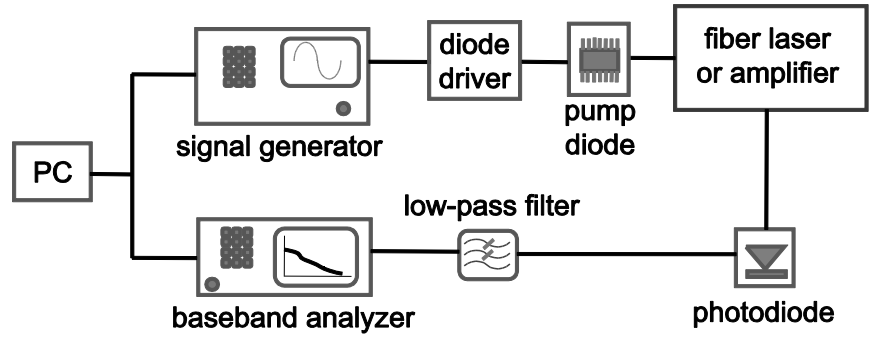

Fig 1. Schematic diagram of the pump modulation transfer setup.

We thoroughly characterize the pump-signal transfer dynamics via measurements of the modulation transfer curves over a range of amplifier parameters and different amplifier configurations. These variations include the seed and pump power levels, the inclusion or omission of the fiber stretcher to suppress the nonlinear effects and the central wavelength of the pulse train $(1030 \mathrm{~nm}$ or $1050 \mathrm{~nm})$. Fig. 2(a) shows a typical curve, obtained for $512 \mu \mathrm{W}$ seed and $100 \mathrm{~mW}$ pump power. The data is fit with a Lorentzian function generalized to allow an arbitrary power coefficient for its frequency dependence: $\xi(v)=\frac{\xi_{0}}{1+\left(v / v_{0}\right)^{n}}$, where $\xi_{0}, v_{0}$ and $n$ are the small-frequency pump-to-signal transfer coefficient, the cut-off frequency, and the power coefficient, respectively. 
(a)
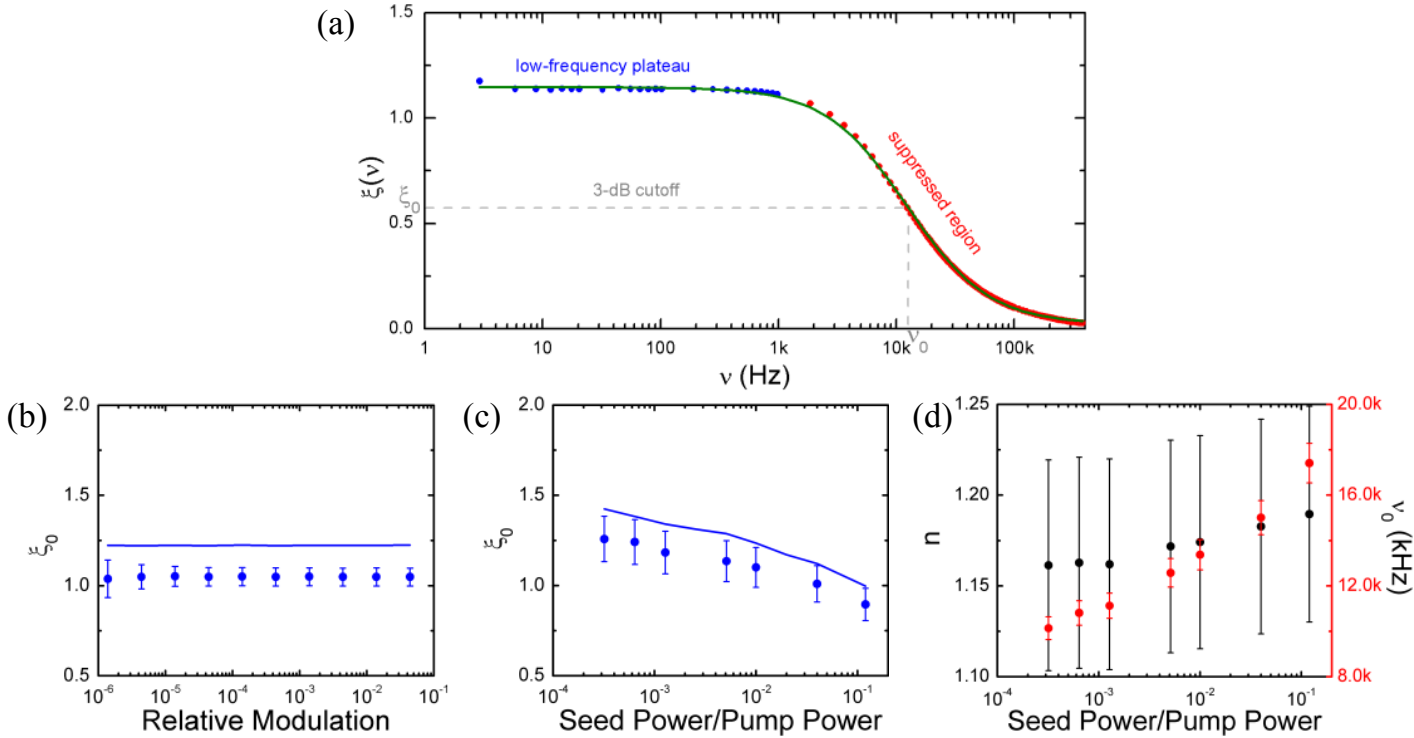

Fig 2. (a) Measured (dots) pump-signal MTF of the pulse fitted with the generalized Lorentzian curve (solid line). The fitting parameters are $\xi_{0}=1.14, n=1.25$ and $v_{0}=12.2 \mathrm{kHz}$. (b) Variation of $\xi_{0}$ with modulation depth; solid line and squares represent the simulated and the experimentally obtained values. (c) Variation of $\xi_{0}$ with the ratio of seed power to pump power; solid line and squares represent the simulated and the calculated values. (d) Variation of $v_{0}$ and $n$ with the ratio of seed power to pump power; solid (dashed) line and upwards triangles (downwards triangles) represent the simulated and the calculated values of $v_{0}(n)$.

The small-frequency transfer coefficient, $\xi_{0}$, is verified to be independent of the modulation depth over a large range of pump power to seed power ratios (Fig. 2(a)). The dependence of the small-frequency transfer coefficient, $v_{0}$, the power coefficient, $n$ and the cutoff frequency, $v_{0}$ on, for example, the ratio of the launched seed power to the pump power are shown in Fig. 2(c) and (d). Thus, Eqn. 1 can be used as a replacement for the actual curve to a very good approximation. The salient features of the experimental dynamics characterized above can be reproduced using the standard three-level gain model. [4,5] We numerically solve this model to obtain the steady-state behavior (i.e., for low modulation frequencies), from which $\xi_{0}$ is determined. Typical results comparing the values obtained by fitting the generalized Lorentzian function to the measured curves and numerically calculated values are shown Fig. 2 (b).

In addition, we seek to utilize the pump-signal MTF information to predict actual amplifier noise using a simplified model for various noise sources. The main contributors to noise are the intrinsic RIN of the seed signal, the contribution of the pump laser RIN and noise created by the quantum fluctuations manifesting as amplified spontaneous emission (ASE). We introduce a simplified approach based on the pump-signal modulation transfer function, where we treat the seed RIN, the RIN transferred from the pump laser and quantum fluctuations to be additive in nature. This approach promptly ignores any interaction between these noise sources. In other words, we assume that all noise sources introduce weak enough fluctuations that they can be treated as first-order perturbations. This is, in practice, comfortably justified in the case for fiber amplification of low-noise mode-locked lasers.

$$
\left\langle I_{\text {amp }}^{2}\right\rangle(v)=\eta_{1}\left\langle I_{\text {seed }}^{2}\right\rangle(v)+\eta_{2}\left\langle I_{\text {pump }}^{2}\right\rangle(v)+\eta_{3}\left\langle I_{A S E}^{2}\right\rangle(v)
$$

Here, $\eta_{1}, \eta_{2}$ and $\eta_{3}$ represent the fractional power of the seed signal, signal added during amplification and ASE, respectively. $\left\langle I_{\text {seed }}^{2}\right\rangle(v)$ and $\left\langle I_{\text {pump }}^{2}\right\rangle(v)$ represent the RIN spectra of the seed pulse train and the pump diode. The quantum fluctuations constitute white noise and given by: $\left\langle I_{A S E}^{2}\right\rangle(v)=\frac{\left\langle I_{A S E}\right\rangle^{2}}{\Delta v_{o p t}}$, where $\Delta v_{\text {opt }}$ is the optical bandwidth of the ASE spectrum. [6] The ASE spectrum is calculated using the same 3-level model. The noise transferred from the pump diode is given by: $\left\langle I_{\text {pump }}^{2}\right\rangle(v)=\left\langle I_{\text {diode }}^{2}\right\rangle(v) \frac{\xi_{0}}{1+\left(v / v_{0}\right)^{n}}$.

For experimental confirmation, we characterize the RIN of fiber-amplified pulses as a function of seed and pump power. In order to visualize the dependence of total noise on seed and pump power levels, contour plots of these quantities are shown for seed wavelengths of $1030 \mathrm{~nm}$ and $1050 \mathrm{~nm}$ in Fig. 3 (a) and (b), respectively. The general topology is as expected: at low seed powers and high pump powers, there is significant ASE generation, which 
increases the overall RIN level. The integrated RIN is observed to decrease linearly with exponentially decreasing seed under constant pump power. We note that the RIN of the launched pulse train and the pump diode are same for $1030 \mathrm{~nm}$ and $1050 \mathrm{~nm}$ seeds. Thus, the differences arise from different amounts of ASE generation simply reflecting the fact that higher seed powers are required to fully saturate the amplifier at $1050 \mathrm{~nm}$, compared to 1030 due to the lower transition cross section of the $\mathrm{Yb}$-doped fiber at the former wavelength.
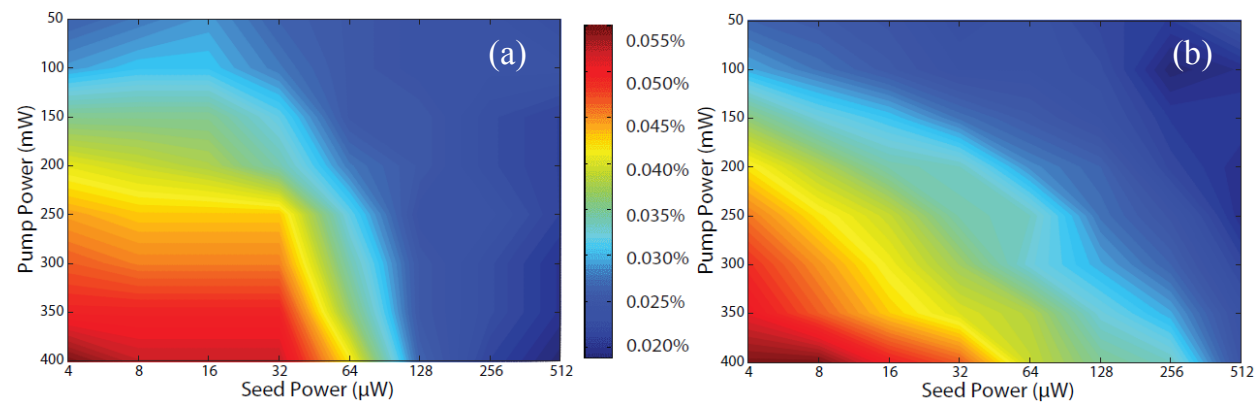

Fig 3. Contour plot of the measured, integrated RIN of the amplified pulse train as a function of seed and pump power levels for seed pulses at central wavelength of (a) $1030 \mathrm{~nm}$ and (b) $1050 \mathrm{~nm}$.

We quantitatively predict the RIN of an amplified pulse train based on measured RIN of the seed source, pump diode and numerically simulated output power and ASE power levels. The RIN of the telecom-grade, 980-nm, singlemode pump laser is measured and found to be independent of its operating power (Figure 4 (a)). The measured and calculated RIN of the amplified pulse train are compared at the fixed pump power of $400 \mathrm{~mW}$. (Fig. 4 (b)) as a function of seed power. Our measurements reveal that external amplification can indeed be accomplished with very little noise imparted to the pulses provided that ASE generation is prevented by ensuring sufficient seed power is present for given pump power level. For the lowest noise amplification results, corresponding to strong seed power, the RIN of the amplifier output is virtually the same as that of the seed source. The integrated RIN of the seed source and amplifier output are $0.015 \%$ and $0.019 \%$ respectively; over the frequency range $1 \mathrm{kHz}-250 \mathrm{kHz}$.
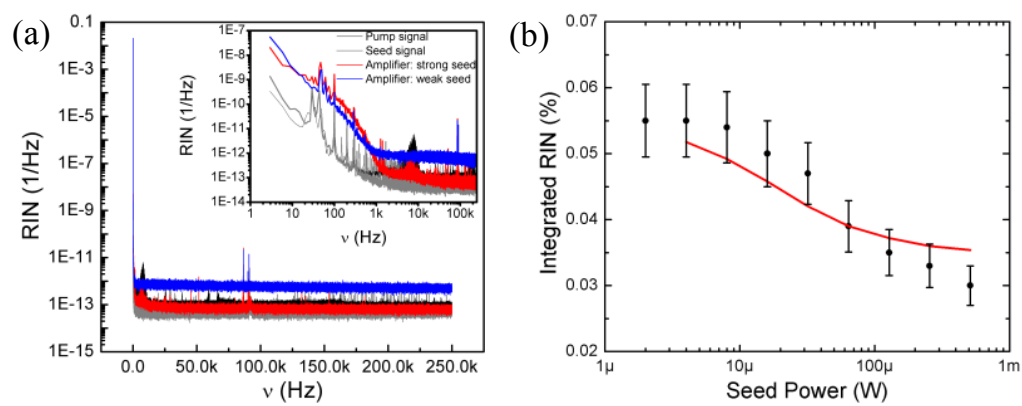

Fig 4. (a) RIN spectra of pump diode, seed source and the amplified pulse train (seed power $12 \mathrm{~mW}$ and pump power of $100 \mathrm{~mW}$ ). (b) Variation of integrated RIN as a function of seed power; solid line (circles) denote calculated (measured) values.

In conclusion, we have characterized the pump-to-signal transfer process for Yb-fiber amplifiers. For low-frequency modulations, the transfer is frequency-independent and the ratio of the signal and pump modulation amplitudes are near unity. At high frequencies, there is a roll off, which means that effectively modulation bandwidths are limited to less than $20 \mathrm{kHz}$ for Yb-fiber amplifiers. This corresponds to an effective upper limit for the bandwidth of carrierenvelop phase stabilization loops acting through modulation of the pump power. Based on the pump modulation transfer information, a simple theoretical model is introduced, that allows one to estimate easily the RIN of the amplified pulse train.

\section{Bibliography}

[1] I. L. Budunoğlu, C. Ülgüdür, Bulent Oktem, and F. Ö. Ilday, "Intensity noise of mode-locked fiber lasers,” Opt. Lett. 34, 2516 (2009).

[2] R. P. Scott, C. Langrock, and B. H. Kolner, "High dynamic range laser amplitude and phase noise measurement techniques," IEEE J. Sel. Top. Quantum Electron. 7, 641 (2001).

[3] T. D. Mulder, R. P. Scott, B. H. Kolner, "Amplitude and envelope phase noise of a modelocked laser predicted from its noise transfer function and the pump noise power spectrum", Opt. Express 16, 14186 (2008).

[4] C. R. Giles and E. Desurvire, "Modeling erbium-doped fiber amplifiers," J. Lightwave Technol. 9, 271 (1991).

[5] R. Paschotta, J. Nilsson, A. C. Tropper, and D. C. Hanna, "Ytterbium-doped fiber amplifiers," IEEE J. Quant. Electron. 33, 1049 (1997).

[6] H. Hodara, "Statistics of thermal and laser radiation", Proc. IEEE 53, 696 (1965). 